\title{
Determining Certain Position and Momentum of a Particle from Uncertainty Principle
}

\author{
Muhammad Yasin \\ Savar, Dhaka, Bangladesh \\ e-mail : md.yasin@ymcontents.com
}

\begin{abstract}
This article will show you how to measure the position and momentum of a particle simultaneously from the principle of uncertainty .From the principle of position-momentum uncertainty, the position and momentum of a particle can be determined at the same time by fulfilling the condition of uncertainty by multiplying a constant $\mathrm{k}$ with the fixed value of position and momentum. Which is known as the principle of certainty.
\end{abstract}

Keywords : uncertainty principle , quantum certainty mechanics , quantum measurement, simultaneous measurement, certain measurement.

Introduction: Knowing the velocity of an object by Newton's law of motion, it is possible to accurately measure the position of the object after time t. That idea was rejected in the quantum world after Heisenberg's discovery of the principle of uncertainty. Since the discovery of the uncertainty principle, we all know that it is not possible to measure the position and momentum of a particle at the same time. But the method of measuring the position and momentum of a particle simultaneously is hidden in the principle of uncertainty. In 1927, Heisenberg invented the principle of uncertainty. The essence of this principle is that "it is not possible to accurately measure the position and momentum of a particle at the same time". The more accurately you know the position, the more uncertain the momentum will be. Again, the more accurate the momentum is, the more uncertain the position will be. had been opposed to the principle of uncertainty since its inception. He was in conflict with Nils Bohr until his death. Einstein would say that surely God does not play dice. Many think Einstein was wrong then. But surprisingly, Einstein and Heisenberg were both right. I have previously published a research paper on quantum certainty mechanics for the relationship between the principle of certainty and the principle of uncertainty. This research paper deals with the method of determining the position and momentum of a particle simultaneously from the principle of uncertainty. The position will be uncertain if the momentum is measured accurately according to the uncertainty principle. Again, if the position is measured with certainty, the momentum will be uncertain. The value of any one of the principles of uncertainty must be uncertain. we take the momentum-position as a packet and multiply an additional $\mathrm{k}$ indefinite value, we will be able to determine the position and momentum of the particle.

Method: Suppose we have two numbers $\mathrm{p}$ and $\mathrm{q}$. We do not know the exact value of $\mathrm{p}$ and $\mathrm{q}$ but we assume that the value of $\mathrm{p}$ and $\mathrm{q}$ is 10 or greater than 10.The higher the value of $\mathrm{p}$ and $\mathrm{q}$, the more accurate $p$ and $q$ will be but there is no set value. To say more value can be infinite value But then there will be problems. So we assume for the convenience of calculation that if the 
value of $\mathrm{p}$ and $\mathrm{q}$ is close to 10 then it will be close to the correct value. Now we are given an equation. That is the product of $\mathrm{p}$ and $\mathrm{q}$ is 10 i.e. $\mathrm{pq}=10$.From this equation, if we get closer to the correct value of $\mathrm{p}$, we will move away from the correct value of $\mathrm{q}$. Again, if we get close to the correct value of $\mathrm{q}$, we will move away from the correct value of $\mathrm{p}$. That is, if you know one value, the value of the other will be wrong. The value of the product of $p$ and $q$ can be 10 in many ways,

$\mathrm{pq}=0$

$10 \times 1=10$

or , $5 \times 2=10$

or, $1 \times 10=10$

or , $2 \times 5=10$

Our equation is,

$\mathrm{pq}=10$

From this equation we will never get the value of $p=10$ and the value of $q=10$ at the same time. But if we multiply an additional constant by pq then the values of $p$ and $q$ will be 10 at the same time. Suppose the correct value of $\mathrm{p}$ is 10 and the correct value of $\mathrm{q}$ is 10 . Then $\mathrm{pqk}=10$ according to the above equation. That is, if $\mathrm{p}=10, \mathrm{q}=10$ then $\mathrm{k}=1 / 10$. Now if the exact value of $\mathrm{p}$ and $\mathrm{q}$ is close to 10 then the value of $\mathrm{k}$ will go towards uncertainty. If we consider $\mathrm{p}$ and $\mathrm{q}$ as a fixed value then the value of $\mathrm{k}$ will be uncertain. According to the above equation, the product of two packets should be 10, where pq is a packet of fixed value and the remaining indefinite value $\mathrm{k}$ is another packet.

(pq)k 10

In this way we will express Heisenberg's principle of uncertainty. According to uncertainty the values of momentum $\mathrm{p}$ and position $\mathrm{x}$ can never be measured with certainty at the same time. If the momentum is confirmed the position will become uncertain. The indefinite product of the two states will be equal to or greater than the Planck constant that is $\Delta \mathrm{p} \Delta \mathrm{x}=\hbar$. Now, according to the uncertainty principle, even if one has a value, one has to be uncertain. Now suppose by any means we know $\Delta \mathrm{p}$ and $\Delta \mathrm{x}$ for sure. Whether by observing it or in some other way. Suppose we were able to invent an instrument that could accurately measure the position $(\Delta x)$ and momentum $(\Delta \mathrm{p})$ of a particle. Then an additional constant $\mathrm{k}$ has to be multiplied to protect the uncertainty principle. $\mathrm{k}$ is an ongoing uncertainty value that has been used to protect the uncertainty principle. If $\Delta \mathrm{x} \Delta \mathrm{p}$ is confirmed then the constant $\mathrm{k}$ will be an uncertain value. Then we can write the equation,

$\Delta \mathrm{p} \Delta \mathrm{xk}=\hbar$

$\mathrm{k}$ is an unknown constant. If $\mathrm{k}=1$ then the uncertainty formula will be $\Delta \mathrm{x} \Delta \mathrm{p}=\hbar$. The definite value of momentum and position has been taken as a packet.

Then the equation will be,

$(\Delta \mathrm{x} \Delta \mathrm{p}) \times \mathrm{k}=\hbar$.

One packet is of momentum-position and the other packet is of constant $\mathrm{k}$.

Now if $\mathrm{k}$ is confirmed $(\Delta \mathrm{x} \Delta \mathrm{p})$ will be uncertain. That is, if $\mathrm{k}=1$ is confirmed $(\Delta \Delta \mathrm{p})$ will be uncertain. Again, if $(\Delta x \Delta p)$ is confirmed, $\mathrm{k}$ will be uncertain. Now suppose, we are sure $(\Delta \mathrm{x} \Delta \mathrm{p})$ then the value of $\mathrm{k}$ is uncertain according to uncertainty.

From $(\Delta \mathrm{x} \Delta \mathrm{p}) \times \mathrm{k}=\hbar$ the uncertainty of energy and time will be:- 
We know kinetic energy $\mathrm{E}=\frac{1}{2} \mathrm{mv}^{2}$.

Now We get by Taking the first derivative of the total $\mathrm{E}$ (i. e. kinetic energy) with respect $\mathrm{p}$, $\Delta \mathrm{E}=\frac{p}{m} \Delta \mathrm{p}$

$\Delta \mathrm{E}=\mathrm{v} \Delta \mathrm{p}$

We get from Equation 25 and Equation 24,

\section{$\Delta \mathrm{E} \Delta \mathrm{xk}=\mathrm{v} \hbar$}

$=>(\Delta \mathrm{E} \Delta \mathrm{t}) \mathrm{k}=\hbar$

From the above equation 26 we can see that imagining energy-time $(\mathrm{E} \Delta \mathrm{t})$ as a fixed packet will make the k constant uncertain.

Equation 24 is divided by Equation 25.

$$
\frac{(\Delta \mathrm{x} \Delta \mathrm{p}) \times \mathrm{k}=\hbar}{(\Delta \mathrm{E} \Delta \mathrm{t}) \mathrm{k}=\hbar}
$$

In equation $27(\Delta \mathrm{x} \Delta \mathrm{p})$ and $(\Delta \mathrm{E} \Delta \mathrm{t})$ are definite values . so we can write,

$$
\frac{(\mathrm{xp}) \times \mathrm{k}=\hbar}{(\mathrm{E} \Delta \mathrm{t}) \mathrm{k}=\hbar}
$$

Since Equation 28 comes from Equation 24, the value of $\Delta \mathrm{k}$ is the same in both cases.

So we can write,

$\mathrm{xp}=\mathrm{Et}$

Therefore,

$\mathrm{xp}=\mathrm{hft}$

Equation 29 is the principle of certainty which comes from the principle of uncertainty.

From equation 29 position $(\mathrm{x})$, momentum $(\mathrm{p})$, time $(\mathrm{t})$, frequency $(\mathrm{f})$ are the definite values. Thus, knowing the momentum and frequency, the position can be determined after $(\mathrm{t})$ time. The more accurately the momentum can be measured, the more certain the position will be.

Decision: $\mathrm{xp}=\mathrm{hft}$ is the principle of certainty by which after knowing the frequency of the waves involved in the electron if we know the momentum we will be able to know the position, and if we know the position we will be able to know the momentum. When we go to see the particle in the laboratory and hit it with a photon, the particle receives hf amount of energy. If we ignore that, we will not be able to know the position and momentum of the particle at the same time. Electrons are particles like a marble(toy) and photons are also particles like a marble(toy).If you hit the marble with the marble, the speed-position is known by calculating the strength of the hit. Similarly, when looking at an electron in a laboratory, it is important to bring in the hf amount of energy in the calculation due to the $\mathrm{F}$ force of the photon. In the above equation hf amount of energy is brought And the momentum-position of the particle can be known simultaneously after the $\mathrm{t}$ time from the equation. $\mathrm{xp}=\mathrm{hft}$; this equation is established by maintaining the condition of uncertainty from the principle of uncertainty. 
Conclusion : The principle of uncertainty is an important formula of quantum mechanics.

The uncertainty principle is $100 \%$ accurate but the momentum and position of the particle can be known with certainty using some conditions in the uncertainty principle. If we know the momentum and the absorbed energy by the particles in the laboratory, we will be able to determine the exact position of the particles after time $t$.From the principle of uncertainty, we add an additional $\mathrm{k}$ constant to the momentum-position uncertainty and energy-time uncertainty of the particle. Then the position and momentum of the particle can be measured at the same time from that equation. And this is the principle of certainty. There is a relationship between uncertainty principle and certainty principle. The principle of uncertainty and the principle of certainty complement each other. I have previously published a quantum certainty mechanics research paper where the principle of uncertainty can be proved from the principle of certainty.

\section{Reference:}

[1] Yasin ,M . Quantum Certainty Mechanics (2021) osf.oi

[2] Ozawa, M: Heisenberg's Original Derivation of the Uncertainty Principle and its Universally Valid Reformulations (2015).arXiv:1507.02010

[3] Busch , P: Heisenberg's uncertainty principle (2007). arXiv:quant-ph/0609185v3

[4] W. Heisenberg, Remarks on the origin of the relations of uncertainty, in: W. Price, S. Chissick(Eds.), The Uncertainty Principle and Foundations of Quantum Mechanics. A Fifty Years'Survey, J. Wiley \& Sons, London, 1977, pp. 3-6 .

[5] W. Heisenberg, Uber den anschaulichen Inhalt der quantentheoretischen $\mathrm{Ki}$ " nematik und Mechanik, Z. Phys. 43 (1927) 172-198.

[6] P. Busch, C.R. Shilladay, Complementarity and uncertainty in Mach-Zehnder interferometry and beyond, Phys. Rep. 435 (2006) 1-31.

[7] Planck M 1900 Zur Theorie des Gesetzes der Energieverteilung im NormalspectrumVerhandlungen der Deutschen Physikalischen Gesellschaft2, 237-45,English translation by D. ter Haar 1967The Old Quantum Theory(PergamonPress]:

[8] https://en.wikipedia.org/wiki/Uncertainty_principle.

[9] Heisenberg, W. The physical content of quantum kinematics and mechanics. In Wheeler, J. A. \& Zurek, W. H. (eds.) Quantum Theory and Measurement, 62-84 (Princeton UP, Princeton, NJ.

[10]Ozawa, M. Noise and disturbance in quantum measurements and operations. Proc. SPIE 6244, 62440Q (2006). 